\title{
Impact of the SARS-CoV-2 (COVID19) pandemic on the morbidity and mortality of high risk patients undergoing surgery: a non-inferiority retrospective observational study
}

Marta Caballero-Milán 1,2,3*, Maria J. Colomina ${ }^{1,2,3^{*}}$, Leo A. Marin-Carcey', Laura Viguera-Fernandez'

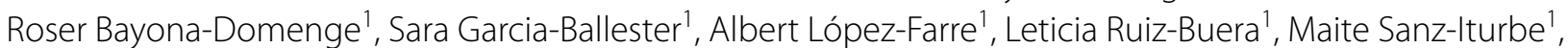
David Álvarez-Villegas ${ }^{1}$, Ely C. Jenssen-Paz ${ }^{1}$, Guillermo Puig-Sanz ${ }^{1}$, Aaron Arcos-Terrones ${ }^{1}$, Carmen Belmonte-Cuenca', Elia Perelada-Alonso ${ }^{1}$, Francho Blasco-Blasco ${ }^{1}$ and Antoni Sabaté ${ }^{1}$

\begin{abstract}
Background: During the COVID-19 crisis it was necessary to generate a specific care network and reconvert operating rooms to attend emergency and high-acuity patients undergoing complex surgery. The aim of this study is to classify postoperative complications and mortality and to assess the impact that the COVID-19 pandemic may have had on the results.

Methods: this is a non-inferiority retrospective observational study. Two different groups of surgical patients were created: Pre-pandemic COVID and Pandemic COVID. Severity of illness was rated according to the Diagnosis-related Groups (DRG) score. Comparisons were made between groups and between DRG severity score-matched samples. Non-inferiority was set at up to $10 \%$ difference for grade III to V complications according to the Clavien-Dindo classification, and up to $2 \%$ difference in mortality.

Results: A total of 1649 patients in the PreCOVID group and 763 patients in the COVID group were analysed; 371 patients were matched for DRG severity score 3-4 (236 preCOVID and 135 COVID). No differences were found in relation to re-operation ( $22.5 \%$ vs. $21.5 \%$ ) or late admission to critical care unit ( $5.1 \%$ vs. $4.5 \%$ ). Clavien grade III to V complications occurred in 107 patients (45.3\%) in the PreCOVID group and in 56 patients (41.5\%) in the COVID group, and mortality was $12.7 \%$ and $12.6 \%$, respectively. During the pandemic, $3 \%$ of patients tested positive for Covid-19 on PCR: 12 patients undergoing elective surgery and 11 emergency surgery; there were 5 deaths, 3 of which were due to respiratory failure following Covid-19-induced pneumonia.
\end{abstract}

Conclusions: Although this study has some limitations, it has shown the non-inferiority of surgical outcomes during the COVID pandemic, and indicates that resuming elective surgery is safe.

\footnotetext{
*Correspondence: marta.caballero@bellvitgehospital.cat

${ }^{1}$ Department of Anaesthesiology and Critical Care, Bellvitge University

Hospital, University of Barcelona, Hospitalet de Llobregat, carrer Feixa Llarga s/n, 08907 Barcelona, Spain

Full list of author information is available at the end of the article
}

(c) The Author(s) 2021. Open Access This article is licensed under a Creative Commons Attribution 4.0 International License, which permits use, sharing, adaptation, distribution and reproduction in any medium or format, as long as you give appropriate credit to the original author(s) and the source, provide a link to the Creative Commons licence, and indicate if changes were made. The images or other third party material in this article are included in the article's Creative Commons licence, unless indicated otherwise in a credit line to the material. If material is not included in the article's Creative Commons licence and your intended use is not permitted by statutory regulation or exceeds the permitted use, you will need to obtain permission directly from the copyright holder. To view a copy of this licence, visit http://creativecommons.org/licenses/by/4.0/. The Creative Commons Public Domain Dedication waiver (http://creativeco mmons.org/publicdomain/zero/1.0/) applies to the data made available in this article, unless otherwise stated in a credit line to the data. 
Trial registration: Clinicaltrials.gov identifier: NCT04780594.

Keywords: Elective surgery, Emergent surgery, SARS-CoV-2 (COVID19) pandemic, Clavien-Dindo complications, Mortality

\section{Introduction}

In December 2019, the disease produced by a new SARS-CoV-2coronavirus, named COVID-19 (Coronavirus Disease 2019), was detected in Wuhan (Hubei Province, China). Since then, the disease has spread rapidly worldwide and was declared a pandemic by the WHO in March 2020.

Spain is among the countries with the highest rate of infection. The latest reports at the end of the first wave of the pandemic (24 May 2020) refer to a total of 235,290 confirmed cases, and a high percentage of hospitalisations for both conventional and critical care.

During the peak of the COVID-19 health emergency, from 11 to 2020 to 15 May 2020, the ratio of patients admitted to hospital was nearly 200 per million population; consequently the number of beds dedicated to COVID-19 infected patients increased. In various wards (Pneumology, Internal Medicine and Infectious Medicine), the highest number of COVID-19 patients admitted in one day during the pandemic was 396, and the number of critical care beds reserved for COVID19 patients increased to 108 .

Low and intermediate elective surgeries were postponed during the pandemic, but high-acuity cases were not, so it was necessary to create a specific care network to attend to non-COVID-19 patients requiring emergency care, critical care, and major/complex surgery, and reconvert operating rooms into critical care beds. This re-organization allowed hospitals to continue with emergency surgical procedures and major oncological and cardiovascular surgery procedures in patients at risk of decompensation. In this context, triage may benefit time-sensitive outcomes in elective surgery.

Various authors have reported that during the pandemic, mortality among COVID-19-infected patients was higher than the rate associated with the same surgical procedures in the pre-pandemic period $[1,2]$. Our working hypothesis, however, is that outcomes in our hospital have not differed in patients operated during vs. before the pandemic. The aim of this study is to evaluate the complications and mortality in patients operated during the COVID crisis. We sought to demonstrate that the pandemic had no negative impact on surgical outcomes in terms of morbidity and mortality when protective protocols for both patients and health care personal were implemented.
We performed this non-inferiority study in healthcare outcomes by comparing 2 periods, pre-pandemic and pandemic. This comparison is also intended to determine quality of healthcare in the operating room. The analysis of our data will help us determine which measures are essential in future COVID-19 crises.

\section{Methods}

\section{Ethics}

Ethical approval for this study ( ${ }^{\circ}$ PR227/20) was provided by the Ethical Committee of Bellvitge University Hospital, Hospitalet de Llobregat, Barcelona, Spain (Chairperson Prof E. Sospedra) on 25 June 2020. Patients were asked to sign an informed consent form to use their data at the time of hospital admission.

\section{Study design}

This is a non-inferiority retrospective observational study. Once the first wave had passed, we decided to analyse the impact that the COVID-19 pandemic had had on our surgical program and on postoperative outcomes. To this end, surgical activity during the pandemic period was compared with a similar period prior to the pandemic.

We also intend to highlight the role of the anaesthesiologists in planning the surgical schedule, selecting patients, and adapting critical care bed capacity to meet surgical needs during the pandemic.

\section{Inclusion criteria}

Using automatically generated data from the minimum data set for surgical procedures that includes all surgical patients (elective and emergent cases), 2 different groups of surgical patients were created:

- Pre-pandemic group (PreCOVID): All patients who underwent surgery from 13 January to 29 February 2020 , far enough from removed from the pandemic to avoid including COVID-19-infected patients.

- Pandemic group (COVID): All patients who underwent surgery from 11 to 2020 to 15 May 2020, during the first wave of the pandemic.

\section{Exclusion criteria}

1. Minor to intermediate surgery performed in the prepandemic period that involves discharge from the hospital on the same day or the following day. 
2. Procedures related to medical treatment or complications of COVID-19 patients, such as chest tubes, extracorporeal oxygenation or tracheostomy.

3. Surgery scheduled during the COVID period in patients whose pre-operative RT-PCR test was positive. In these cases, surgery was postponed until the patients were negative, but they were not eligible for inclusion in the study even if they eventually underwent surgery.

\section{Setting and management in the COVID Period}

Several organisational changes were made to the surgical pathway: the sICU and trauma-ICU were moved to the operating room (OR) area for non-COVID patients, and were reduced from 24 critical beds in the pre-pandemic period to 11 critical beds in the COVID period. Other measures were preoperative online symptomatic screening for COVID in scheduled surgery patients and RT-PCR screening for emergency patients. All spaces in the OR area were clearly differentiated: clean COVID OR, COVID OR, and clean ICU; different entry and exit routes were used for the OR Area and sICU to ensure the operating room area was safe for surgery and postoperative and critical management. A specific COVID OR was used for emergency surgery patients with a positive RT-PCR; after the procedure patients were admitted to a COVID-ICU. An airway management protocol was implemented, and the use of personal protective equipment was mandatory for all staff involved in the OR and sICU OR.

Surgery departments triaged elective surgery patients for high acuity cases, and these were discussed at the weekly planning meeting if Covid-19 screening was negative. The anaesthesiologists coordinated the surgical schedule, and adjusted the critical bed capacity to surgical needs. After surgery, all patients were screening for Covid-19 infection by RT-PCR.

\section{Data collection}

Data on patient demographics, clinical history, surgical team and surgical procedure, primary and secondary diagnoses, and primary and secondary surgical procedures were collected automatically. A case-mix grouping system based on diagnosis related groups using ICD-10-AM International Classification of Diseases nomenclature was created. The Severity of Illness Index assigns to each patient an overall severity score (from 1 to 4 ) and mortality risk score (from 1 to 4). [3].
The primary end point was the percentage of patients with grade III to V complications according to the Clavien-Dindo classification.

The relevant variables analysed for all patients included in both periods were the following:

- Age.

- Sex.

- Severity according to the DRG scale.

- Risk according to the DRG scale.

- Day of admission to the hospital.

- Day of surgery.

- Diagnosis of the pathology.

- Type of surgical intervention by specialty and timing (scheduled or emergency).

- Per-protocol admission to the sICU (scheduled surgery).

- Late, non-scheduled admission to sICU.

- Length of hospital stay.

- Positive RT-PCR screening for Infection by COVID19.

- Surgical complications according to the Clavien Dindo classification.

- In-hospital mortality.

- Discharge destination (home, death, or nursing home).

Data were collected from the administrative database in November 2020, so all patients were followed up until they were discharged from the hospital. Additionally, in patients with high risk or DRG severity score (3 and 4) and emergent procedures the full clinical history was analysed and the cause of death was determined by consensus between the main study investigators (MC, MJC, and AS).

Patients in whom preoperative or postoperative COVID-19 testing resulted positive (diagnosis confirmed by RT-PCR) were also analysed on the basis of their full clinical history. A nosocomial infection was considered if patients manifested COVID-19 symptoms from the third day of admission until discharge.

\section{Sample size}

During the Covid period, a total of 763 patients were operated. In order to determine the size of the sample, we anticipated a $15 \%$ Clavien III-V complication rates among patients during this period and an inclusion ratio of 2:1 with a power of $90 \%$ and a significant alfa level of $5 \%$, resulting in 1527 patients to be analysed from the PreCovid period.

\section{Statistical analysis}

Comparisons were made between the PreCOVID group and the COVID group. In order to avoid bias, a selected 
matched sample on the basis of the DRG severity score was created in both groups and compared. The results were analysed after selecting matched patients.

A descriptive analysis was performed using the usual statistical tests: Chi-square tests to compare the categorical variables, and parametric or non-parametric tests for continuous variables (depending on their normality). For all variables, bilateral tests with a significance level of $5 \%$ were used. Criteria for Non-inferiority were: up to $10 \%$ difference for grade III to V complications according to the ClavienDindo classification, and up to $2 \%$ difference for mortality.

\section{Results}

\section{Participants}

During the COVID period, 21 patients were excluded from the analysis because the procedure performed involved medical patients with COVID-19 infection (pleural procedure, tracheostomy and extracorporeal oxygenation). In total, 2412 patients were analysed during both study periods. In the PreCOVID period, 1649 patients were operated (PreCOVID group) and 763 patients in the COVID group. Four subgroups were created on the basis of the type of surgery:

1. Major scheduled surgery performed during the preCOVID-19 period.

2. Emergency surgery performed during the preCOVID-19 period.

3. Major elective surgery performed during the COVID 19 period.

4. Emergency surgery performed during the COVID-19 period.

Comparisons between the PreCOVID group and COVID group are shown in Table 1 . Most patients in the COVID group were women with greater severity and higher DRG risk scores; more urologic procedures were performed and fewer general and digestive surgeries. Fewer patients in the COVID group were admitted per protocol to the sICU, but more required late admission to the sICU and had higher Clavien-Dindo scores; however, hospital length of stay, the percentage of patients discharged home and non-survivors did not differ (Table 1).

In both periods, 48 patients ( $2 \%)$ died in both groups (additional information in supplementary Tables 1 , access on the website), and severe complications (Clavien grade III to V) occurred in 195 patients (8\%). In a multivariate analysis, deaths correlated with age (odds ratio $0.95,95 \%$ CI $0.924-0.988$ ) and DRG severity score (odds ratio 0.177 , $95 \%$ IC 0.08-0.38), but not with the period when the surgical procedure was performed (odds ratio $1.2195 \%$ IC 0.56-2.6).
Scheduled surgery (groups 1-3, Table 1)

A total of 1490 patients underwent major elective procedures during the pre-COVID period, and 585 patients in the COVID period. The COVID group was similar in age, were mostly women, with greater severity and higher DRG risk scores, and fewer were admitted per protocol to the sICU. There were no differences in mortality and length of hospital stay. The percentage of patients discharged home was similar (Table 1).

\section{Emergency surgery (groups 2-4, Table 1)}

A total of 159 patients underwent emergency surgery during the pre-pandemic period, and 178 patients in the pandemic period. The DRG risk of mortality was higher in the COVID group. Fewer patients in the COVID group were admitted to the sICU. No differences were found in mortality, discharge home, or length of stay.

\section{Matched subgroups according to the DRG severity score (Table 2)}

In a subgroup of patients matched by DRG severity score 3 and 4, (group 5 and 6), 236 patients were considered DRG severity 3-4 in the PreCOVID group and 135 patients in the COVID group. There were no differences between matched groups in terms of age, emergency procedure, and surgical speciality; however, more men were included in the matched pre-pandemic subgroup, and per protocol admission to the sICU was higher in this subgroup. No differences were found in relation to re-operation ( $22.5 \%$ vs. $21.5 \%)$, late admission to sICU ( $5.1 \%$ vs. $4.5 \%$ ), or length of hospital stay. Severe complications (Clavien grade III to V) occurred in 107 patients $(45.3 \% \%)$ in the Pre-COVID group and in 56 patients $(41.5 \%)$ in the COVID group, and mortality was $12.7 \%$ and $12.6 \%$, respectively.

\section{COVID-19}

COVID-19 infection during both periods occurred in 25 patients (Table 3). Two occurred in the pre-pandemic period as a post-operative complication; this was classed as a nosocomial infection.

In the pandemic period, 12 patients undergoing elective surgery tested positive for Covid-19 on RT-PCR in the postoperative period; all survived. Eleven patients undergoing emergency surgery tested positive on RTPCR before surgery; 3 died due to Covid-19-induced pneumonia leading to respiratory failure; 2 other deaths in in this group patients with positive RT-PCR were not related to Covid-19 pneumonia. Overall, 23 patients $(3 \%)$ in the COVID group tested positive on RT-PCR. 
Table 1 Patient demographics and outcomes in both study periods (preCOVID and COVID groups)

\begin{tabular}{|c|c|c|c|c|c|c|c|}
\hline PERIOD & $\begin{array}{l}\text { PRECOVID } \\
(n=1649)\end{array}$ & $\begin{array}{l}\text { PRECOVID } \\
\text { ELECTIVE } \\
(n=1490)\end{array}$ & $\begin{array}{l}\text { PRECOVID } \\
\text { EMERGENT } \\
(n=159)\end{array}$ & $\begin{array}{l}\text { COVID } \\
(n=763)\end{array}$ & $\begin{array}{l}\text { COVID } \\
\text { ELECTIVE } \\
(n=585)\end{array}$ & $\begin{array}{l}\text { COVID } \\
\text { EMERGENT }(n=178)\end{array}$ & $\begin{array}{l}\text { PRECOVID } \\
\text { vs. } \\
\text { COVID ( } p \text { value) }\end{array}$ \\
\hline $\begin{array}{l}\text { Age, years } \\
\text { (IC 95\%) }\end{array}$ & $\begin{array}{l}62.2(61.3- \\
62.7)\end{array}$ & $62(61.3-62.8)$ & $63.7(60.7-66.7)$ & $\begin{array}{l}66.1(64.6- \\
67.7)\end{array}$ & $\begin{array}{l}63.1(61.8- \\
64.4)\end{array}$ & $64.6(61.9-67.4)$ & 0.065 \\
\hline Male sex, n (\%) & $899(54.5)$ & 807 (54.2) & $92(57.9)$ & $379(49.7)$ & $281(48)$ & $98(55.1)$ & 0.028 \\
\hline SPECIALTY & & & & & & & 0.018 \\
\hline $\begin{array}{l}\text { Digestive and } \\
\text { Gynaecological, } \\
\text { n (\%) }\end{array}$ & $461(28)$ & $391(26.2)$ & $70(44)$ & $177(23.2)$ & $125(21.4)$ & $52(29.2)$ & \\
\hline $\begin{array}{l}\text { Orthopaedics } \\
\text { and Trauma, } \\
\text { n (\%) }\end{array}$ & $294(17.8)$ & $264(17.7)$ & 30 (18.9) & $140(18.3)$ & $85(14.5)$ & 55 (30.9) & \\
\hline $\begin{array}{l}\text { Plastic and Neck, } \\
\text { n (\%) }\end{array}$ & $288(17.5)$ & $276(18,5)$ & $12(7.6)$ & 148(19.4) & $130(22.2)$ & $18(10.1)$ & \\
\hline $\begin{array}{l}\text { Cardiovascular } \\
\text { and thoracic, } \\
\mathrm{n}(\%)\end{array}$ & 291 (17.6) & $271(18.2)$ & $20(12.6)$ & $138(18.1)$ & $112(19.2)$ & $26(14.6)$ & \\
\hline $\begin{array}{l}\text { Neurosurgery, } \\
\mathrm{n}(\%)\end{array}$ & $160(9.7)$ & $138(9,3)$ & $22(13.8)$ & $69(9.1)$ & $50(8.5)$ & $19(10.7)$ & \\
\hline Urology, n (\%) & 155 (9.4) & $150(10.1)$ & $5(3.1)$ & 91 (11.9) & $83(14.2)$ & $8(4.5)$ & \\
\hline \multicolumn{8}{|l|}{ DRG SEVERITY } \\
\hline $1-2(\%)$ & $1413(85.7)$ & 1318 (88.5) & 95 (59.7) & $650(82.3)$ & $500(85.5)$ & $128(71.9)$ & 0.123 \\
\hline 3-4 (\%) & $236(14.3)$ & $172(11.5)$ & $64(40.3)$ & $135(17.7)$ & $85(14.5)$ & $50(28.1)$ & 0.034 \\
\hline \multicolumn{8}{|l|}{ DRG RISK } \\
\hline $1-2, n(\%)$ & 1485 (90.1) & 1378 (92.5) & $107(67.3)$ & $650(85.2)$ & 524 (89.6) & $126(70.8)$ & 0.000 \\
\hline $3-4, n(\%)$ & $164(9.9)$ & $113(7.6)$ & $58(36.5)$ & $113(14.8)$ & $61(10.4)$ & $52(29.2)$ & 0.000 \\
\hline $\begin{array}{l}\text { REINTERVEN- } \\
\text { TION, n (\%) }\end{array}$ & $138(8.4)$ & $112(7.5)$ & $26(16.4)$ & $55(7.2)$ & $38(6.5)$ & $17(9.6)$ & 0.375 \\
\hline \multicolumn{8}{|l|}{ SICU ADMISSION } \\
\hline $\begin{array}{l}\text { Per protocol, } \\
\text { n (\%) }\end{array}$ & $470(28.5)$ & $430(28.9)$ & $40(25.1)$ & $130(17)$ & $102(17.4)$ & $28(15.7)$ & 0.000 \\
\hline $\begin{array}{l}\text { Late admission, } \\
\mathrm{n}(\%)\end{array}$ & $20(1.2)$ & $11(0.7)$ & $9(5.6)$ & $18(2.4)$ & $15(2.6)$ & $3(1.7)$ & \\
\hline \multicolumn{8}{|l|}{ CLAVIEN DINDO } \\
\hline I, n (\%) & 1445 (87.6) & 1370(91.8) & $75(44.8)$ & $580(76)$ & $503(85.9)$ & 77 (43.3) & \\
\hline II, n (\%) & $84(5.1)$ & $50(3.4)$ & $34(20.6)$ & $108(14.2)$ & $42(7.2)$ & $66(37.1)$ & \\
\hline Illa, n (\%) & $9(0.5)$ & $4(0.3)$ & $5(3)$ & $6(0.8)$ & $2(0.3)$ & $4(2.2)$ & \\
\hline IIlb, n (\%) & $31(1.9)$ & $20(1.3)$ & $11(6.7)$ & $23(3)$ & $15(2.6)$ & $8(4.5)$ & \\
\hline IVa, n (\%) & $25(1.5)$ & $13(0.9)$ & $12(7.3)$ & $16(2.1)$ & $10(1.7)$ & $6(3.4)$ & \\
\hline $\mathrm{IVb}, \mathrm{n}(\%)$ & $24(1.5)$ & $15(1)$ & $9(5.5)$ & $13(1.7)$ & $8(1.4)$ & $5(2.8)$ & \\
\hline V, n (\%) & $31(1.9)$ & $18(1.3)$ & $13(12.1)$ & $17(2.2)$ & $5(0.9)$ & $12(6.7)$ & \\
\hline III-V & $120(7.3)$ & $70(4.7)$ & $50(31.4)$ & $75(9.8)$ & $40(6.8)$ & $35(19.7)$ & 0.037 \\
\hline $\begin{array}{l}\text { Length of stay } \\
\text { (days) }\end{array}$ & $10(9-11)$ & $9.1(8-10.1)$ & $18.7(14.7-22.7)$ & $9.8(8.3-11.3)$ & $7.6(6.5-8.7)$ & $17(11.5-22.5)$ & 0.774 \\
\hline \multicolumn{8}{|c|}{ DESTINATION AT DISCHARGE } \\
\hline Home (\%) & 63.1 & 70 & 58.5 & 70.9 & 73.1 & 63.5 & \\
\hline Death (\%) & 1.9 & 1.2 & 8.2 & 2.2 & 0.9 & 6.7 & 0.638 \\
\hline $\begin{array}{l}\text { Nursing home } \\
\text { (\%) }\end{array}$ & 353 & 28.8 & 33.3 & 26.9 & 26 & 29.8 & \\
\hline
\end{tabular}


Table 2 Patient demographics and outcomes between matched DRG severity score 3-4

\begin{tabular}{|c|c|c|c|}
\hline PERIOD & PRECOVID GROUP $(n=236)$ & COVID GROUP $(n=135)$ & $\begin{array}{l}\text { PRECOVID } \\
\text { VS COVID } \\
\text { ( } p \text { value) }\end{array}$ \\
\hline Age (years) & $66(64-68)$ & $66(63.5-69)$ & 0.914 \\
\hline Sex, male (\%) & $165(69.9)$ & $75(55,6)$ & 0.007 \\
\hline SURGERY BY SPECIALTY & & & 0.418 \\
\hline Digestive and Gynaecological, n (\%) & $53(22.5)$ & $32(23.7)$ & \\
\hline Orthopaedics and Trauma, n (\%) & $34(14.4)$ & $27(20)$ & \\
\hline Plastic and Neck, n (\%) & $23(9.7)$ & $18(13.3)$ & \\
\hline Cardiovascular and thoracic, n (\%) & $79(33.5)$ & $35(26)$ & \\
\hline Neurosurgery, n (\%) & $29(12.3)$ & $15(11.1)$ & \\
\hline Urology, n (\%) & $18(7.6)$ & $8(5.9)$ & \\
\hline REINTERVENTION (\%) & $53(22.5)$ & $29(21.5)$ & $0-897$ \\
\hline \multicolumn{4}{|l|}{ sICU } \\
\hline Per protocol, n (\%) & $152(64.4)$ & $60(44.4)$ & 0.000 \\
\hline Late admission, n (\%) & $12(5.1)$ & $6(4.4)$ & \\
\hline \multicolumn{4}{|l|}{ Clavien Dindo } \\
\hline l, n (\%) & $67(28.4)$ & $33(24.4)$ & \\
\hline$\|, \mathrm{n}(\%)$ & $62(26.3)$ & $45(33.3)$ & \\
\hline IIla, n (\%) & $8(3.4)$ & $3(2.2)$ & \\
\hline IIIb, n (\%) & $26(11)$ & $15(11.1)$ & \\
\hline Iva, n (\%) & $21(8.9)$ & $11(8.2)$ & \\
\hline IVb, n (\%) & $22(9.3)$ & $11(8.2)$ & \\
\hline V, n (\%) & $30(12.7)$ & $17(12.6)$ & \\
\hline III-V & $107(45.3)$ & $56(41.5)$ & 0.515 \\
\hline Length of stay (days) & 32.7 & 26.5 & 0.774 \\
\hline \multicolumn{4}{|l|}{ DESTINATION AT DISCHARGE } \\
\hline Home (\%) & 45.3 & 48 & \\
\hline Dead (\%) & 12.7 & 12.6 & 1 \\
\hline Others (\%) & 42 & 39.4 & \\
\hline
\end{tabular}

Only the patients with a DRG severity score 3-4 are shown. These are considered the patients with the highest risk of complications. DRG: Diagnosis Related Groups. Chi-square tests to compare the categorical variables, and the subtraction of variables using parametric or non-parametric tests for the continuous variables

\section{Discussion}

Overall, patients in the COVID period presented greater severity and higher DRG risk scores, and emergency surgery was more frequent. They had more Clavien III-V complications, but this difference did not reach the significance required to show inferiority. There were no differences in hospital length of stay in patients discharged home and in-hospital deaths with respect to the Prepandemic period. Death or disability (Clavien-Dindo IV and V) occurred in $4.8 \%$ in the COVID group vs. $6.1 \%$ in the PreCOVID group; this difference was not significant. This percentage in the COVID group is similar to that reported in other studies [4]. However, when comparing GRD severity-matched groups with similar ages and types of surgery (emergency and general surgery), no differences were found in terms of re-operation, ClavienDindo complications, length of hospital stay and mortality. These results show the non-inferiority outcome in the COVID population, despite the limitations imposed by the reorganisation of the surgical pathway, particularly the shortage of sICU beds, during the Covid-19 pandemic.

The reduction of surgical critical care beds during the pandemic period clearly limited the number of major elective surgery procedures performed. However, late sICU admission in high severity matched samples was similar in both periods. In non-epidemic conditions, around $40 \%$ of critical beds are used for trauma and surgical patients [5], but only $13 \%$ are used for elective surgery and around $6 \%$ for patients receiving mechanical ventilation following elective surgical procedures [5]. Based on these figures and our experience, even if the number of critical beds were reduced, rationalising the use of the sICU should minimise the need to suspend elective surgery, and should not be a limitation for scheduling more surgical patients in future Covid-19 waves. 


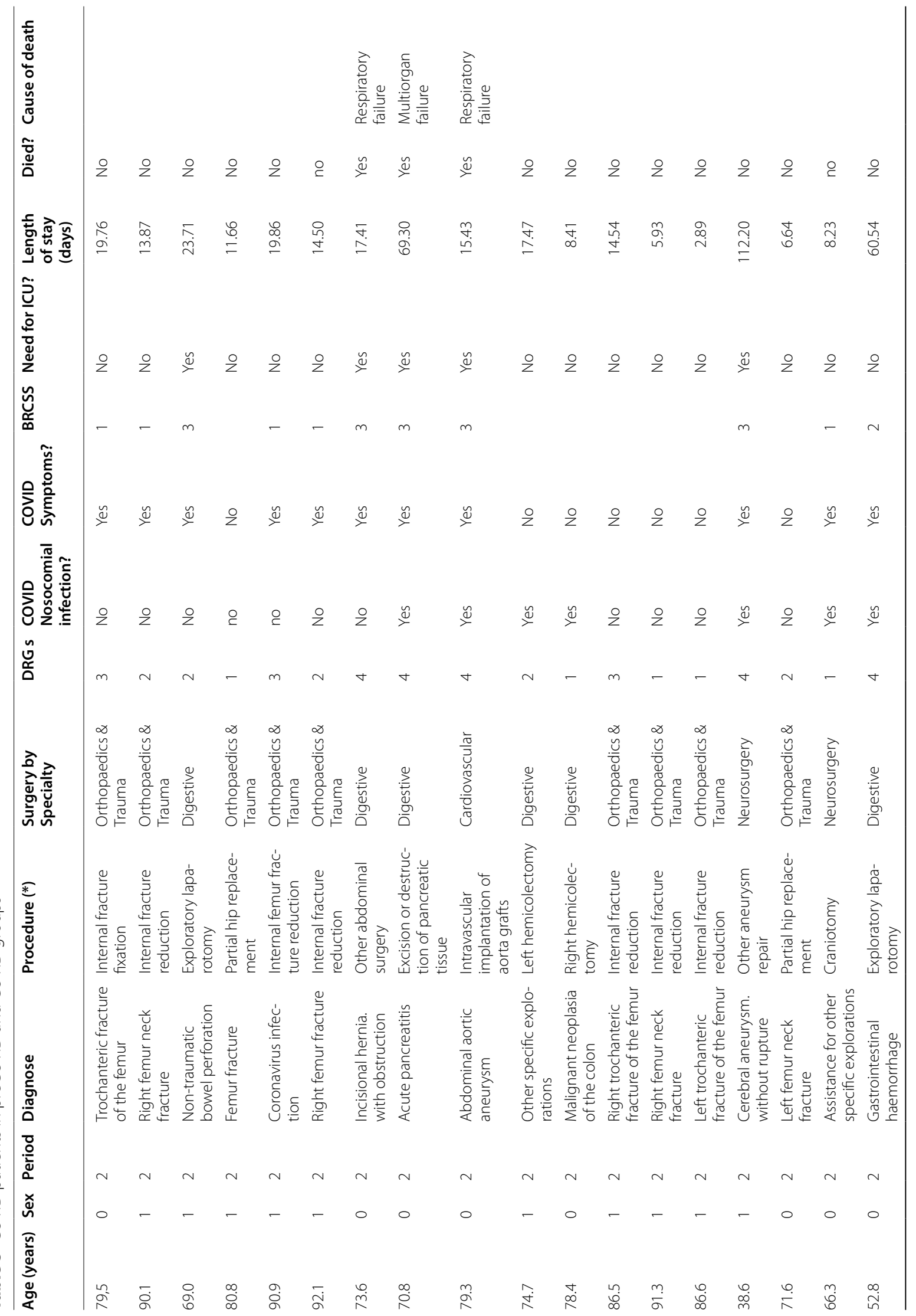




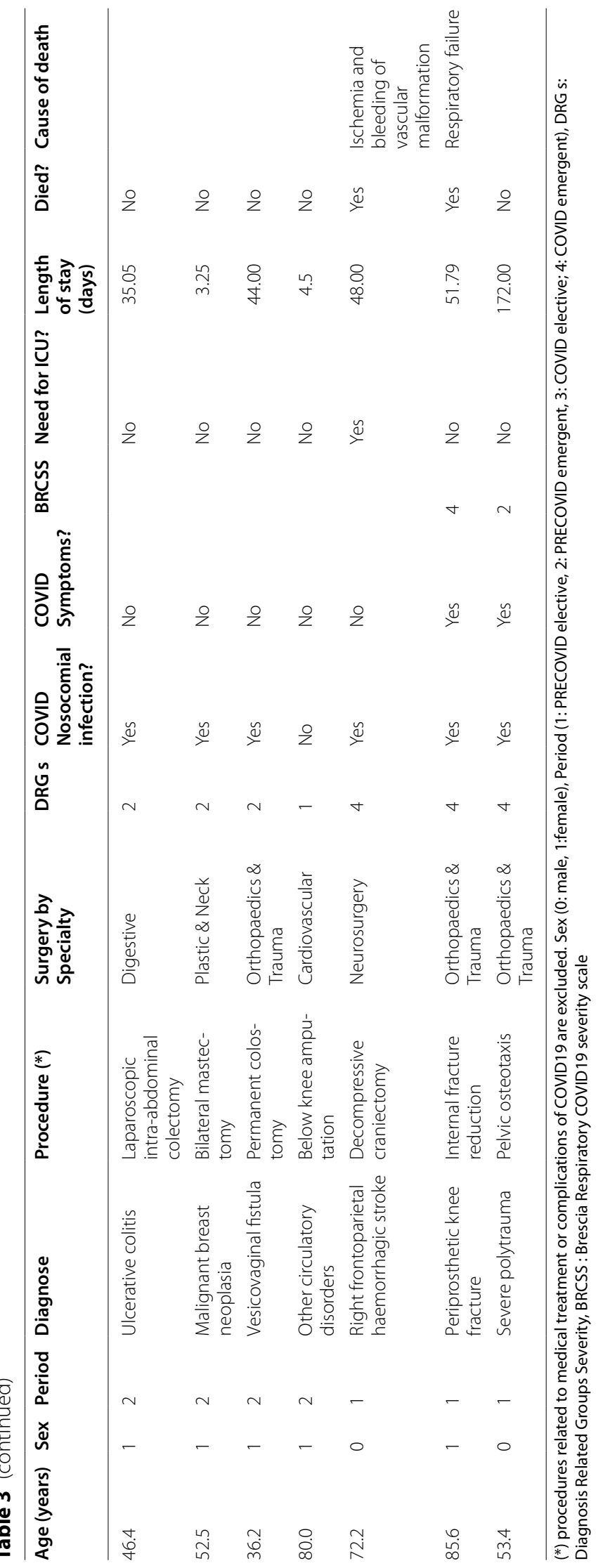


In our cohort, age was associated with mortality in a multivariate analysis, a finding similar to other series. Advanced age predicts a 2- to 4-fold increase in morbidity and mortality [6]. Furthermore, age and pre-existing disease severity are factors for 1-year mortality after an acute episode [7]. In our cohort, DRG severity was associated with mortality. The DRG severity score is an indirect but incomplete measure of frailty, which is defined as "a multidimensional syndrome characterized by decreased reserves that leaves an individual vulnerable to adverse outcomes due to decreased tolerance of stressors (physical, physiologic, or psychosocial)" [8]. The prevalence of frailty is higher in emergency vs. elective procedures [9]. Frailty is consistently associated with an increase in the risk of major morbidity, mortality, and readmissions [10], and increases the odds of non-home discharge among older patients [11].

The re-operation rate was high in both subgroups of patients matched by DRG severity score 3 and 4 . This had a major influence on severe complications (Clavien grade III to V) and mortality, which was $12.7 \%$ and $12.6 \%$ respectively. Efforts should be made to improve surgical outcomes, even in patients with severe medical comorbidity. These results are similar to those of the Collaborative study [2], in which age and emergency surgery were significant predictors of mortality.

In the COVID group, 12 asymptomatic patients tested positive on RT-PCR during hospitalization, which represents $2 \%$ of elective surgeries. These patients were not detected in the preoperative clinical screening for Covid-19, and could potentially spread SARS- CoV-2 to other patients and caregivers. This figure is similar to that reported by Kane et al. [12]. Detecting COVID-19 infected patients is a challenge; RT-PCR may be prone to sampling error and asymptomatic patients may have a lower viral load than symptomatic COVID-19 patients [13]. The benefit of chest computed tomography associated with RT-PCR is limited [14]. Moreover, chest CT is not recommended for screening in asymptomatic patients [15]. Fourteen days of isolation may improve screening of elective surgery patients; however, outliers may need more than 14 days [16].

Patients infected with SARS-CoV-2 have an increased risk of postoperative complications and mortality [1]. Five of the 25 Covid-19 infected patient $(20 \%)$ died. Covid-19 pneumonia was the cause of death in 3 patients from the COVID period. These figure are similar to those reported by other authors. Lei et al. [17] described postoperative outcomes in a group of 34 Covid-19 positive patients undergoing surgery - $44 \%$ required admission to the ICU and $20.5 \%$ died. An international cohort study reported a 30-day in-hospital mortality rate of $19 \%$ in patients undergoing non-emergency surgery who were diagnosed with Covid-19 peri-operatively [2].
Due to protective protocols for both patients and health care personal and RT-PCR screening, no anaesthesiologists or surgical nurses were infected in the operating room during the pandemic period in the rugical theatre, but 3 surgeons had apositive RT-PCR in the same period.

There are some limitations to this study: first, because of its retrospective nature and the heterogeneity of the population, our results cannot be generalized to other hospitals. However, the data were collected automatically, double checked for postoperative complications, and are therefore reliable. Also, the severity and risk scores indicate that any bias introduced is more likely to occur in the COVID group.

\section{Conclusions}

This study has showed the non-inferiority outcome of surgery performed in the COVID period; grade III to $\mathrm{V}$ complications and mortality slightly favours the COVID group. Our study shows that other hospitals can safely resume elective surgery during a pandemic if protective protocols for both patients and health care personal, RT-PCR screening, and 14 days quarantine for elective surgery patients are implemented. In summary, this study indicates that reassuming surgery in a pandemic is safe.

\section{Abbreviations}

SARS-CoV-2: severe acute respiratory syndrome coronavirus 2; COVID19: : coronavirusdisease 2019; COVID: coronavirus disease; DRG: Diagnostic-related groups; WHO: WorldHealth Organization; RT-PCR: REALTIME-polymerase chain reaction; sICU: surgicalintensive care unit; trauma-ICU: trauma-intensive care unit; OR: operatingroom; ICD-10AM: InternationalClassification of Diseases and Related Health Problems, Tenth Revision,Australian Modification.

\section{Supplementary Information}

The online version contains supplementary material available at https://doi. org/10.1186/s12871-021-01495-3.

Additional file 1.

Acknowledgements

We would like to thank Pilar Fernández Rodriguez for providing all data from the administrative database.

\section{Authors' contributions}

MC, MJC, LAM, LV, RB, SG, AL, LR, MS, DA, ECJ, GP, AA, CB, EP, FB, AS have made substantial contributions to the conception and design of the manuscript, the acquisition, analysis and interpretation of data, and either wrote the paper or substantively revised the manuscript. MC, MJC, LAM, LV, RB, SG, AL, LR, MS, $D A, E C J, G P, A A, C B, E P, F B, A S$ read and approved the submitted version (and any substantially modified version that involves the author's contribution to the study). MC, MJC, LAM, LV, RB, SG, AL, LR, MS, DA, ECJ, GP, AA, CB, EP, FB, $A S$ agreed to be personally accountable for the author's contributions, and to ensure that questions related to the accuracy or integrity of any part of the study, even those in which the author was not personally involved, are appropriately investigated, resolved, and the resolution is documented in the literature. 


\section{Funding}

There are no public or private sources of funding for this study.

\section{Availability of data and materials}

All study data is supplied in the tables attached to this article and in the supplementary material table. Please contact with the corresponding author (MC) if some data is requested.

\section{Declarations}

\section{Ethics approval and consent to participate}

Ethical approval for this study ( $N^{\circ}$ PR227/20) was provided by the Ethical Committee of Bellvitge University Hospital, Hospitalet de Llobregat, Barcelona, Spain (Chairperson Prof E. Sospedra) on 25 June 2020. Patients were asked to sign an informed consent form to use their data at the time of hospital admission admittance.

All methods were performed in accordance with the relevant guidelines and regulations.

\section{Consent for publication}

All authors of this manuscript give their consent for publication.

\section{Competing interests}

The authors declare that they have no conflict of interest.

\section{Author details}

'Department of Anaesthesiology and Critical Care, Bellvitge University Hospital, University of Barcelona, Hospitalet de Llobregat, carrer Feixa Llarga s/n, 08907 Barcelona, Spain. ${ }^{2}$ Health Group, Neuroscience Program, Perioperative Physiopathology and Pain, Institut d'Investigació Biomèdica de Bellvitge IDIBELL, Hospitalet de Llobregat, Barcelona, Spain. ${ }^{3}$ Department of Clinical Sciences, School of Medicine, Universitat de Barcelona - UB, L'hospitalet de Llobregat (Barcelina), Barcelona, Spain.

Received: 3 May 2021 Accepted: 22 October 2021

Published online: 26 November 2021

\section{References}

1. Aminian A, Safari S, Razeghian-Jahromi A, Ghorbani M, Delaney CP. COVID-19 outbreak and surgical practice: unexpected fatality in perioperative period. Ann Surg. 2020;272:e27-9.

2. Nepogodiev D, Glasbey JC, Li E, et al. COVIDSurg Collaborative. Mortality and pulmonary complications in patients undergoing surgery with perioperative SARS-CoV-2 infection: an international cohort study. Lancet. 2020;396:27-38

3. De Marco MF, Lorenzoni L, Addari P, Nante N. Evaluation of the capacity of the APR-DRG classification system to predict hospital mortality Epidemiol Prev. 2002;26:183-90.

4. Kasivisvanathana V, Lindsaya J, Rakshani-Moghadama S, Elhamshary A, Kapriniotis K, Kazantzis G. A cohort study of 30 day mortality after NONEMERGENCY surgery in a COVID-19 cold site. Int J Surg. 2020;84:57-65.

5. Poeran J, Zhong H, Wilson L, Liu J, Memtsoudis SG. Cancellation of Elective Surgery and Intensive Care Unit Capacity in New York State: A Retrospective Cohort Analysis. Anesth Analg. 2020;131:1337-4.

6. Turrentine FE, Wang H, Simpson VB, Jones RS. Surgical risk factors, morbidity, and mortality in elderly patients. J Am Coll Surg. 2006;203:865-77.

7. Shankar-Hari M, Saha R, Wilson J, et al. Rate and risk factors for rehospitalisation in sepsis survivors: systematic review and meta-analysis. Intensive Care Med. 2020;46:619-36.

8. Fried LP, Ferrucci L, Darer J, Williamson JD, Anderson G. Untangling the concepts of disability, frailty, and comorbidity: implications for improved targeting and care. J Gerontol A Biol Sci Med Sci. 2004;59:255-63.

9. Mclsaac DI, Bryson GL, van Walraven C. Association of frailty and 1-year postoperative mortality following major elective noncardiac surgery: a Population-Based Cohort Study. JAMA Surg. 2016;151:538-45.

10. Mclsaac DI, Taljaard M, Bryson GL, et al. Frailty as a predictor of death or new disability after surgery: a Prospective Cohort Study. Ann Surg. 2020;271:283-9.
11. Mclsaac DI, Beaulé PE, Bryson GL, Van Walraven C. The impact of frailty on outcomes and healthcare resource usage after total joint arthroplasty: a population-based cohort study. Bone Joint J. 2016;98:799-805.

12. Kane AD, Paterson J, Pokhrel S, et al. Peri-operative COVID-19 infection in urgent elective surgery during a pandemic surge period: a retrospective observational cohort study. Anaesthesia. 2020;75:1596-604.

13. Liu R, Han H, Liu F, et al. Positive rate of RT-PCR detection of SARS-CoV-2 infection in 4880 cases from one hospital in Wuhan, China, from Jan to Feb 2020. Clin Chim Acta. 2020;505:172-5.

14. Puylaert, on behalf of the SCOUT study group. Yield of Screening for COVID-19 in Asymptomatic Patients Before Elective or Emergency Surgery Using Chest CT and RT-PCR (SCOUT). Multicenter Study. Ann Surg. 2020;272:919-24.

15. Rubin GD, Haramati LB, Kanne JP, et al. The role of chest imaging in patient management during the COVID-19 pandemic: a multinational consensus statement from the Fleischner society. Radiology. 2020;S00123692. https://doi.org/10.1148/radiol.2020201365.18.

16. Linton NM, Kobayashi T, Yang Y, et al. Incubation period and other epidemiological characteristics of 2019 novel coronavirus infections with right truncation: a statistical analysis of publicly available case data. J Clin Med. 2020;9:538

17. Lei S, Jiang F, Su W, et al. Clinical characteristics and outcomes of patients undergoing surgeries during the incubation period of COVID-19 infection. EClinicalMedicine. 2020;21:100331.

\section{Publisher's Note}

Springer Nature remains neutral with regard to jurisdictional claims in published maps and institutional affiliations.

Ready to submit your research? Choose BMC and benefit from:

- fast, convenient online submission

- thorough peer review by experienced researchers in your field

- rapid publication on acceptance

- support for research data, including large and complex data types

- gold Open Access which fosters wider collaboration and increased citations

- maximum visibility for your research: over 100M website views per year

At BMC, research is always in progress.

Learn more biomedcentral.com/submissions 